\title{
Karlsruhe Nuclide Chart - New 10th edition 2018
}

\author{
Zsolt Sóti ${ }^{1, *}$, Joseph Magill ${ }^{2}$, and Raymond Dreher ${ }^{2}$ \\ ${ }^{1}$ European Commission, Joint Research Centre (JRC), PO Box 2340, 76125 Karlsruhe, Germany \\ 2 Nucleonica GmbH, Magdeburger Str. 2, 76139 Karlsruhe, Germany
}

Received: 20 November 2018 / Received in final form: 12 February 2019 / Accepted: 2 April 2019

\begin{abstract}
Obtaining nuclear data is an international activity with new and updated data constantly being determined by thousands of scientists at major research centres worldwide. Because of the large amounts of data generated and the formats used to store these data, the field of nuclear data is highly specialised. To make the most important key data more accessible to a wider audience, nuclide charts have been developed. In this article, we present the scientific highlights of the new 10th Edition of the Karlsruhe Nuclide Chart. The main focus of this Chart is to provide structured, accurate information on the half-lives and decay modes, as well as energies of the emitted radiation for over 4000 experimentally observed ground states and isomer nuclides to an interdisciplinary audience.
\end{abstract}

\section{Introduction}

The United Nations has designated 2019 as the international year of the periodic table of chemical elements, recognizing it as an instrument "central to linking cultural, economic and political aspects of the global society through a common language". First introduced in 1869 by Dmitry Mendeleev, the periodic table currently features 118 elements, identified by the number of protons in their atomic nucleus. Variants of a given element differing in the number of neutrons are called isotopes, most of which are produced artificially and are unstable (radioisotopes). The study of isotope properties, synthesis, and decay modes has been instrumental for rationalizing phenomena, providing a basis for our understanding of the Universe, and resulted in a plethora of applications with deep societal and economic impact. It is thanks to our understanding of isotopes that we can power spacecraft for exploring the cosmos, elaborate sophisticated climate change models, deploy accurate environmental control systems, apply powerful diagnostic and therapeutic tools for fighting diseases, etc.

Today, more than 4000 isotopes (more generally known as nuclides) are known and can be characterized and classified in an extended form of the periodic table known as a nuclide chart. In this article, we describe in detail the latest edition of the Karlsruhe Nuclide Chart (KNC).

\footnotetext{
* e-mail: zsolt.soti@ec.europa.eu
}

The new 10th Edition of the Karlsruhe Nuclide Chart contains nuclear data on 4040 experimentally observed nuclide ground states and isomers. New data on 696 nuclides has been included together with information on 47 new nuclides. Since the previous edition, IUPAC has agreed on names for the elements 113, 115, 117, and 118 (nihonium, symbol Nh, moscovium Mc, tennessine Ts and oganesson Og, respectively).

In addition to half-lives, decay modes, and energies of the emitted radiations, most recent data on atomic weights, isotopic abundancies, cross sections and thermal fission yields (for U 235 and $\mathrm{Pu} 239$ ) are given. Together with a fold-out nuclide chart, a 72 page booklet gives additional information on important physical constants, properties of the chemical elements and more than 80 socalled reduced decay schemes to assist in the understanding of the nuclide box contents. In the following sections, these features are described in more detail.

\section{The structure of the Karlsruhe Nuclide Chart}

In contrast to the periodic table, a nuclide chart is based on the number of protons and neutrons in the nucleus of the atom. A nuclide chart is a two dimensional representation of the nuclear and radioactive properties of all known atoms. A nuclide is the generic name for atoms characterized by the constituent protons and neutrons. In contrast to the periodic table which is based on chemical behaviour, the nuclide chart arranges nuclides according to the number of protons and neutrons in the nucleus. Each 


\section{The Karlsruhe Nuclide Chart}

A nuclide chart is a two dimensional representation of the nuclear and radioactive properties of all known atoms. A nuclide is the generic name for atoms characterized by the constituent protons and neutrons. The nuclide chart arranges nuclides according to the number of protons (vertical axis) and neutrons (horizontal axis) in the nucleus. Each nuclide in the chart is represented by a box containing the element symbol and mass number, half-life, decay types and decay energies, etc.

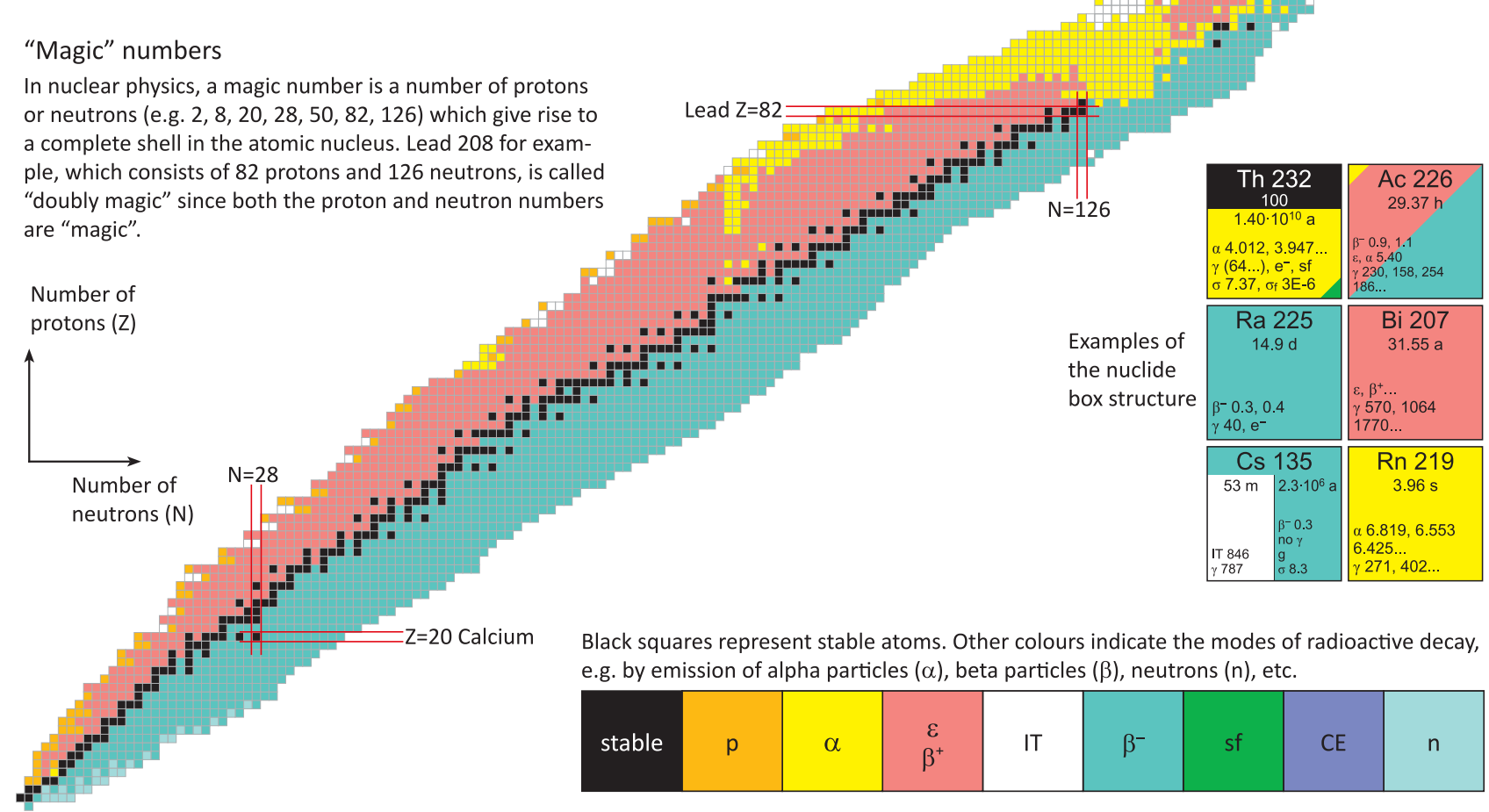

Fig. 1. Schematic diagram of the Karlsruhe Nuclide Chart showing the main features.

nuclide in the chart is represented by a box containing the element symbol and mass number, natural abundance, half-life, decay types and decay energies. The nuclide boxes are coloured according to their decay properties. Nuclear isomers, or excited states, can be represented by subdividing the box accordingly.

The Karlsruhe Nuclide Chart is shown schematically in Figure 1. The horizontal axis corresponds to the neutron number $(\mathrm{N})$ and the vertical axis to the proton number $(\mathrm{Z})$ in the atomic nucleus. Each horizontal line of nuclide boxes (constant Z, variable $\mathrm{N}$ ) corresponds to the isotopes of a chemical element. With coordinates $(\mathrm{N}, \mathrm{Z})$ the coloured nuclide box shows data for experimentally observed nuclides with $\mathrm{N}$ neutrons and $\mathrm{Z}$ protons in the nucleus. A nuclide box contains the nuclide name, mass number (total number of neutrons and protons $\mathrm{N}+\mathrm{Z}$ ), the half-life for radioactive nuclides or atomic abundance for stable nuclides, data on decay modes and radiations. Data in an undivided box, as shown in Figure 1, corresponds to the ground state of the nuclide. If a box is divided into two or three sections (Fig. 1 Cs 135) the right-most section corresponds to the ground state: box sub-sections to the left of the ground state section represent isomers. Usually, all known isomers are shown in the chart. If, however, an isomer decays by isomeric transition (IT), only isomers with half-lives greater than or equal $1 \mathrm{~s}$ are shown. In some cases, isomers with sub-second half-lives are shown if they are deemed to be important.
Stable nuclides are shown in black, unstable with colours representing the decay modes. As can be seen in Figure 1, the stable nuclides (black boxes) are located in the centre of the nuclide chart. For the low mass numbers (low $\mathrm{N}+\mathrm{Z}$ ), the nucleus contains almost equal numbers of protons and neutrons. At higher mass numbers, because of the coulomb force of positively charged protons, a higher neutron number per proton is necessary for stability.

The colour coding used in the nuclide boxes indicates the decay modes. Blue is for $\beta^{-}$decay (Fig. 1 Ra 225) which occurs when there is an excess of neutrons in the nucleus. Red is for electron capture or $\beta^{+}$decay $\left(\varepsilon / \beta^{+}\right)$(Fig. $1 \mathrm{Bi} 207$ ) which occurs when there is an excess of protons in the nucleus. Yellow is for alpha decay (Fig. 1 Rn 219) which is a characteristic of high mass number nuclides. In addition, light blue is used for neutron decay, light brown for proton decay, green for spontaneous fission, violet for cluster decay or cluster emission and white for isomeric transition. Multiple decay modes for a single nuclide are represented by differently sized triangles. A box divided diagonally into two large triangles implies that two decay modes have been observed both with branching ratios in the range 5-95\% (Fig. 1 Ac 226). A small coloured triangle in the corner of a nuclide box denotes a decay branching ratio less than or equal to $5 \%$ (Fig. 1 Th 232). Primordial nuclides are those which were created during the formation of terrestrial matter and are still present on Earth because of their very long half-lives. In the Karlsruhe Nuclide Chart, such nuclides have both an 


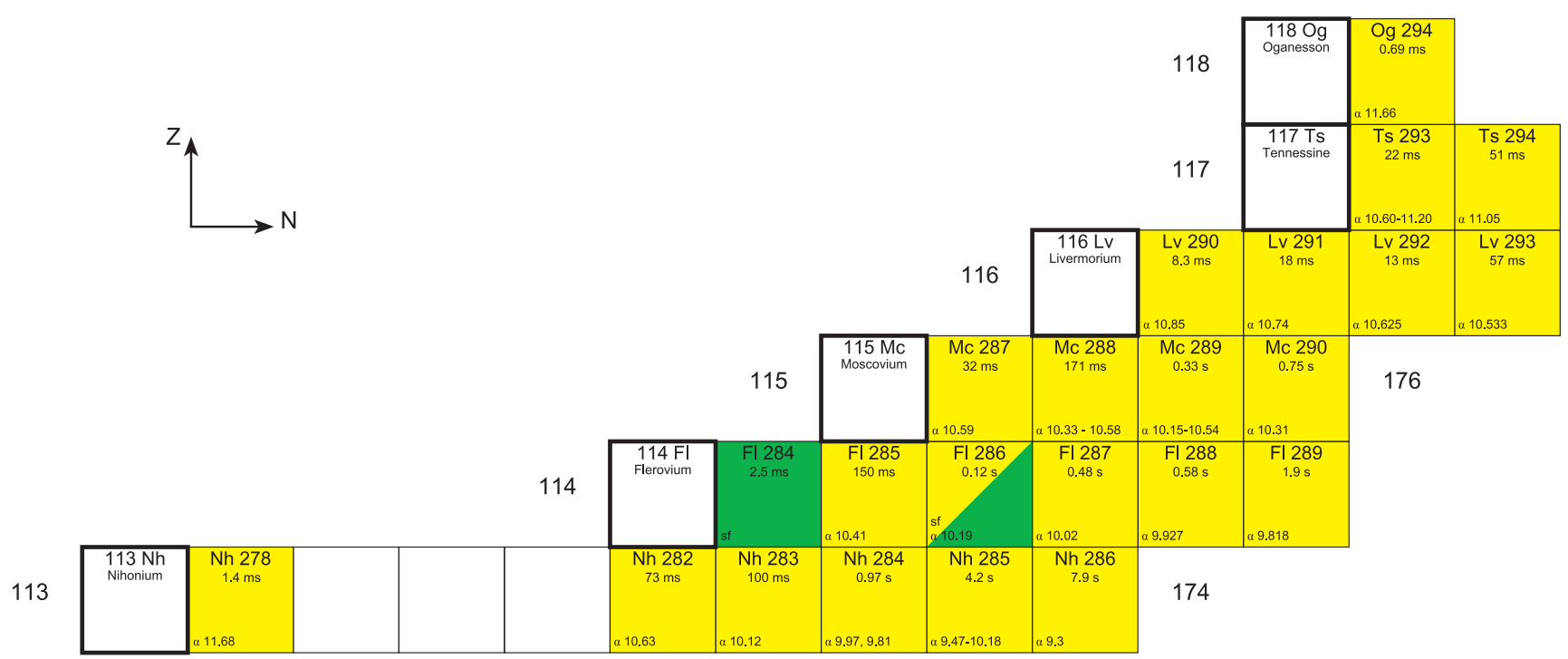

Fig. 2. New symbols for chemical elements in the Karlsruhe Nuclide Chart. Symbol Nh in line 113, Mc in 115 , Ts in 117 and Og in 118.

Table 1. The 47 newly discovered nuclides and isomers in the 10th edition of the Karlsruhe Nuclide Chart.

\begin{tabular}{|c|c|c|c|c|c|c|c|}
\hline F $26 \mathrm{~m}$ & $\mathrm{Na} 17$ & $\mathrm{Cl} 29 \mathrm{~m}$ & $\mathrm{Cl} 38 \mathrm{~m}$ & Ar 30 & Ar $30 \mathrm{~m}$ & Mn 73 & Fe 76 \\
\hline Co $72 \mathrm{~m}$ & Co 78 & $\mathrm{Ni} 54 \mathrm{~m}$ & Ni 81 & Ni 82 & $\mathrm{Cu} 83$ & $\mathrm{Ga} 80 \mathrm{~m}$ & Ge 59 \\
\hline Se 63 & Kr 67 & Kr 68 & $\mathrm{Pd} 90$ & Ag 92 & Cd 94 & In 96 & $\mathrm{Ba} 130 \mathrm{~m}$ \\
\hline Ba 154 & La 155 & La 156 & Ce 157 & Ce 158 & $\operatorname{Pr} 158$ & $\operatorname{Pr} 159$ & $\operatorname{Pr} 160$ \\
\hline Nd 162 & $\mathrm{Pm} 139 \mathrm{~m}$ & Sm 166 & Dy 170 & $\mathrm{~Pb} 178$ & Th $229 \mathrm{~m}$ & U 221 & Np 219 \\
\hline Np 223 & Am 223 & Am 229 & Bk 233 & Bk 236 & Es 240 & Fl 284 & \\
\hline
\end{tabular}

isotopic abundance and an experimentally observed half-life. In the Chart they are indicated in the black upper section of the nuclide box containing information on the atomic abundance. The lower section of the nuclide box indicates the decay mode and the half-life (Fig. 1 Th 232).

\section{Updates in the new 10th Edition}

\subsection{New names of chemical elements}

In 2016, IUPAC announced the names of elements 113, 115, 117 and 118 to be nihonium (Nh), moscovium (Mc), tennessine (Ts), and oganesson (Og) [1]. Nihonium was proposed by the RIKEN Nishina Center for AcceleratorBased Science (Japan); moscovium and tennessine were proposed by the Joint Institute for Nuclear Research, Dubna (Russia), Oak Ridge National Laboratory (USA), Vanderbilt University (USA) and Lawrence Livermore National Laboratory (USA). Oganesson was proposed by the Joint Institute for Nuclear Research, Dubna (Russia) and Lawrence Livermore National Laboratory (USA) in recognition of Prof. Yuri Oganessian, a pioneer in the field. The new element names are shown in the 10th edition of the Chart (Fig. 2).

\subsection{New decay and radiation data}

The new 10th edition of the "Karlsruher Nuklidkarte" presents decay data on 4040 experimentally observed ground state and isomer nuclides. In total, 649 nuclides were updated. The most important sources of the decay and radiation data were nuclide mass evaluations in the Nuclear Data Sheets (NDS) [2] and the Evaluated Nuclear Structure Data File (ENSDF) [3]. The update procedure is described in detail in Section 3.4. The 47 new nuclides, not found in the previous (2015) [4] edition, were added.

In Figure 3, the highlighted coloured boxes represent new and updated nuclides in the 2018 edition. As can be seen, many of the updated nuclides lie on an inclined line indicating that they were updated using new mass evaluations from the Nuclear Data Sheets (each new mass evaluation gives rise to a line in the figure). Updated (blue) nuclides in the region around $\mathrm{Z}=60, \mathrm{~N}=105$ were the result of a major scientific investigation in 2017.

The 47 newly discovered nuclides and isomers reported in the 10th edition of the Karlsruhe Nuclide Chart are shown in Table 1.

\subsection{Reduced decay schemes}

Due to the limited space available in each nuclide box, only the most important or key nuclear data can be inserted. There are also a number of rules which determine exactly how this data is inserted. It is important to know how the inserted data should be interpreted. For this reason, socalled reduced decay schemes have been developed for many nuclides to help in the understanding of the condensed form used in the nuclide boxes. The "reduced" refers to the fact that full decay scheme has been simplified 


\section{Karlsruhe Nuclide Chart Online, KNCO++}

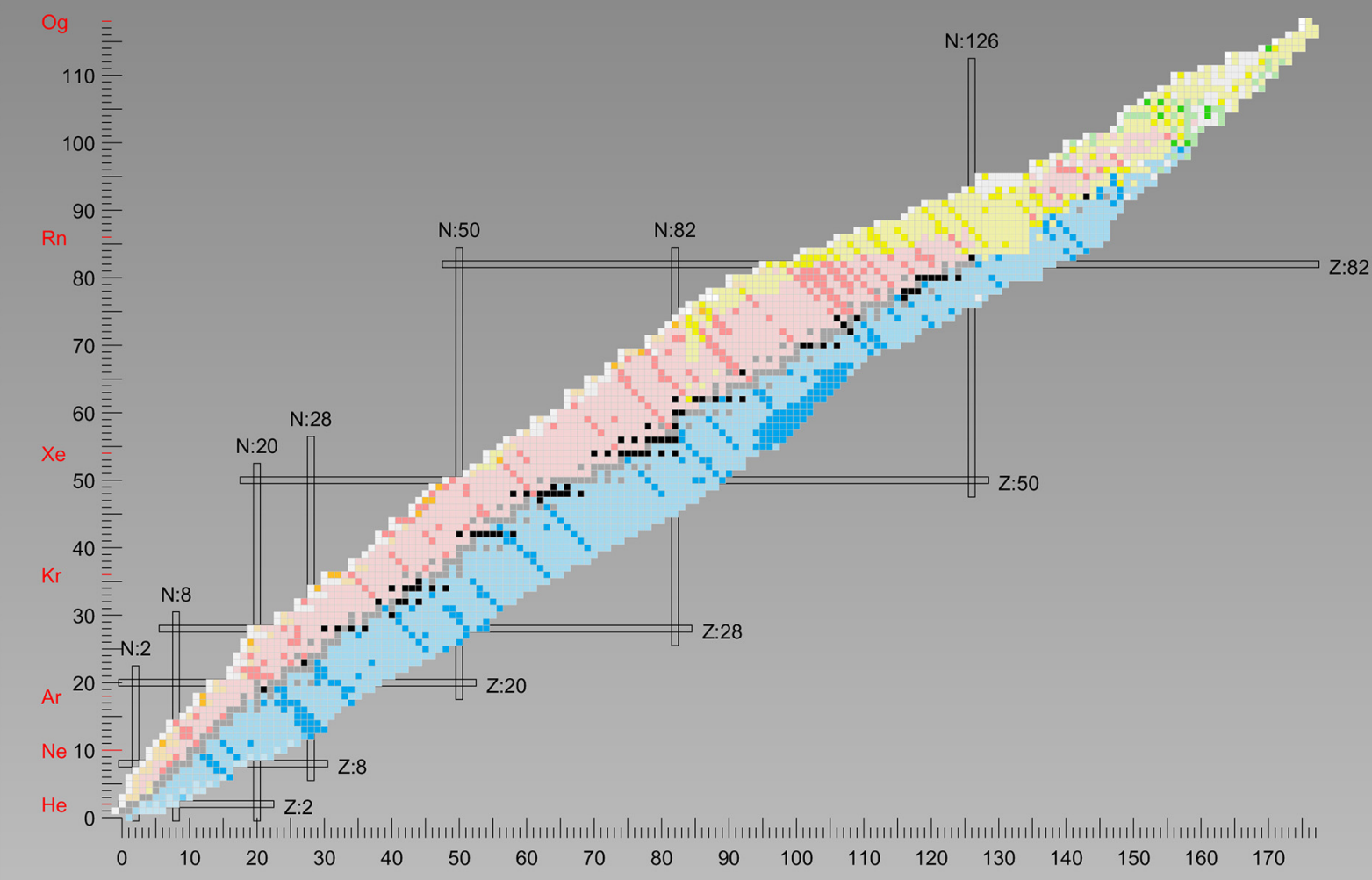

Fig. 3. The "Difference" Chart. The coloured boxes indicate new and updated nuclides in the 10th edition. The difference chart was created using the online version of the Karlsruhe Nuclide Chart $(\mathrm{KNCO}++)$ available in the nuclear science web portal Nucleonica https://nucleonica.com/.

to concentrate on the essential features. In the booklet accompanying the Chart, more than 80 such decay schemes have been included.

In this section, an example of a nuclide box and accompanying reduced decay scheme as shown in Figures 4a and 4b for Cs 134 is described in detail. Transitions which are not indicated in the nuclide box, but which have been added to improve understanding, are shown as dotted lines. The corresponding data is shown in grey. Radiation energies followed by dots in the nuclide box (indicating a low emission probability) are shown in the decay scheme with dotted lines.

In Figures $4 \mathrm{a}$ and $4 \mathrm{~b}$ it can be seen that Cs 134 has a ground state with a half-life of 2.0652 years and a metastable state Cs $134 \mathrm{~m}$ with a half-life of $2.912 \mathrm{~h}$.

\subsubsection{Decay of the ground state Cs 134}

The blue colour of the nuclide box indicates that the ground state decays by $\beta^{-}$emissions. The dots following the beta particle endpoint energy $(0.7 \ldots)$ indicate several $\beta^{-}$particles with different endpoint energies. The beta transitions are shown in more detail in the reduced decay scheme in Figure $4 \mathrm{~b}$. In the nuclide box, only the beta emission with highest emission probability is shown $(0.7 \mathrm{MeV})$. This value has been rounded as can be seen from Figure $4 \mathrm{~b}$.

The blue arrows indicate that the ground state Cs 134 decays by $\beta^{-}$emissions. Cs 134 is characterised by the emission of several $\beta^{-}$particles with different endpoint energies. As can be seen from the decay scheme, the $0.658 \mathrm{MeV}$ transition is followed by the emission of 796 and $605 \mathrm{keV}$ gamma photons to the ground state Ba 134. Additional gamma emissions shown in the decay scheme are indicated by dots in the nuclide box (i.e. $\gamma 605,796 \ldots$ ).

The small red triangle in the top left corner and the $\varepsilon$ in the nuclide box imply that a weak electron capture occurs followed by $847 \mathrm{keV}$ gamma emission to the ground state Xe 134 (positron emission has not been observed even though the energy difference between the parent and daughter nuclide is more than $1.022 \mathrm{MeV}$, which is the threshold for this type of decay). 


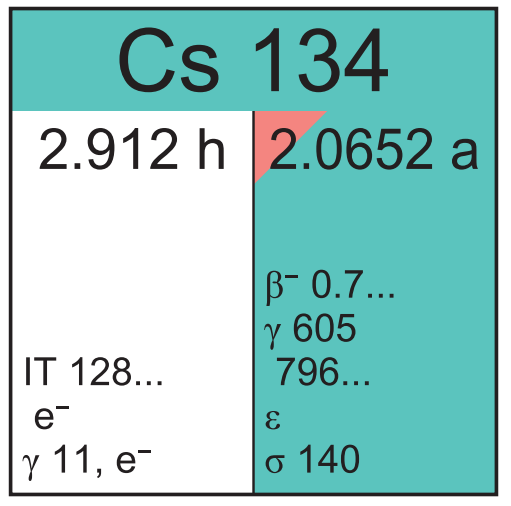

(a)

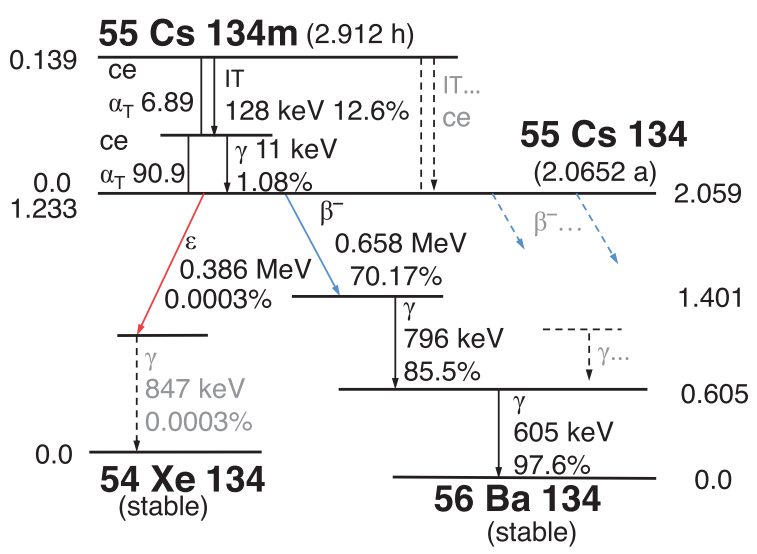

(b)

Fig. 4. Nuclide box (a) and the reduced decay scheme (b) for Cs 134 and Cs $134 \mathrm{~m}$.

The last row in the box, i.e. $\sigma 140$, gives the $(n, \gamma)$ cross section for the thermal neutrons in barn for the formation of Cs 135 .

\subsubsection{Decay of the metastable state Cs $134 \mathrm{~m}$}

The metastable state Cs $134 \mathrm{~m}$, with half-life $2.912 \mathrm{~h}$, decays by isomeric transition (IT) indicated by the colour white in the nuclide box. In the nuclide box, the entry IT $128 \ldots$ indicates that the most probable transition energy is $128 \mathrm{keV}$. This energy can be dissipated either through the competing processes of gamma emission or though the emission of conversion electrons (indicated in the nuclide box by $\mathrm{e}^{-}$). This is shown in more detail in the reduced decay scheme in Figure 4b. The so-called total conversion coefficient $\alpha_{\mathrm{T}}$, which is the ratio of conversion electron to gamma emission, has a value of 6.98 indicating that conversion electron emission is predominant. The $128 \mathrm{keV}$, (mainly conversion electron) emission to an excited state of Cs 134, further de-excites through an $11 \mathrm{keV}$ transition to the ground state $\mathrm{Cs} 134$. This $11 \mathrm{keV}$ transition is also highly "converted" $\left(\alpha_{\mathrm{T}}=90.9\right)$ such that again conversion electron emission is dominant.

\subsection{Sources of decay data and the update procedure}

Updating the Karlsruhe Nuclide Chart for a new edition is a continuous process based on the following main tasks:

- information and decay data on recently discovered nuclides is obtained from original scientific papers;

- for regular updates of radioactive decay data for existing nuclides in the KNC, the main sources of data are the Nuclear Data Sheets (NDS), and the Evaluated Nuclear Structure Data File (ENSDF);

- for the creation and updating of reduced decay schemes for individual nuclides, the latest data from NDS, ENSDF, and NUBASE2016 [5] are used;

- creation and maintenance of scientific references for each added/updated nuclide.

In this section these processes are described in more detail.

\subsubsection{Recently discovered nuclides}

Information on recently discovered nuclides is found through periodic evaluations of original papers published in scientific journals. Typically the following journals are consulted: Physical Review C, Physical Review Letters, Physics Letters B, Nuclear Physics A, The European Physical Journal A, Nature, Radiochimica Acta and Nuclear Instruments and Methods. Since the 7th edition from 2006, more than 660 new ground state and isomer nuclides have been identified and added to the Chart.

\subsubsection{Regular updates of radioactive decay data for existing nuclides}

The Nuclear Data Sheets (NDS) are published as articles on a monthly basis based on the Evaluated Nuclear Structure Data File (ENSDF). In each article one or more mass evaluations are presented. This evaluated decay data is then used to update the Karlsruhe Nuclide Chart using the values for the half-life, branching ratios, particle and photon emission energies and probabilities.

If a nuclide in the evaluated mass chain has a daughter product with a different mass number (resulting, for example, from the emission of alpha, proton, neutron, etc.) then the ENSDF database is consulted to find the most recent NDS evaluations of the daughter product. Alternatively, it may contain information on new evaluations which are not yet published.

The branching ratios for decay modes are important since they determine the use of small or large triangles in the nuclide boxes (see the inset in Fig. 1). Small coloured triangles indicate a branching ratio of $\leq 5 \%$, whereas large triangles indicate a branching ratio in the range $5-95 \%$. Data on the branching ratios are given in NDS. If the referenced data is older than that given in NUBASE2016, then the latter data is used.

\subsubsection{Creation and updating of reduced decay schemes}

In the reduced decay scheme diagrams given in the booklet, data on Q-values and energy levels are shown. The Q-values are taken from the NUBASE2016 or NDS 


\title{
Am 241
}

\section{Adopted values 10th edition}

\author{
$\mathrm{J}^{\pi}$ : \\ $5 / 2-$
}

Half-life:

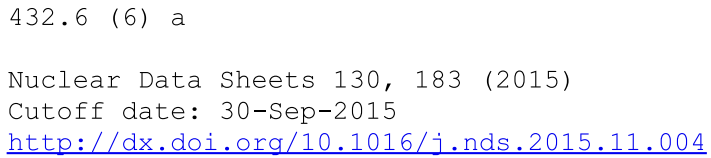

Decay mode:

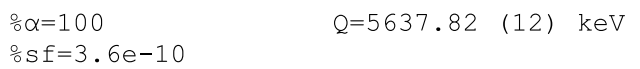

Radiation:

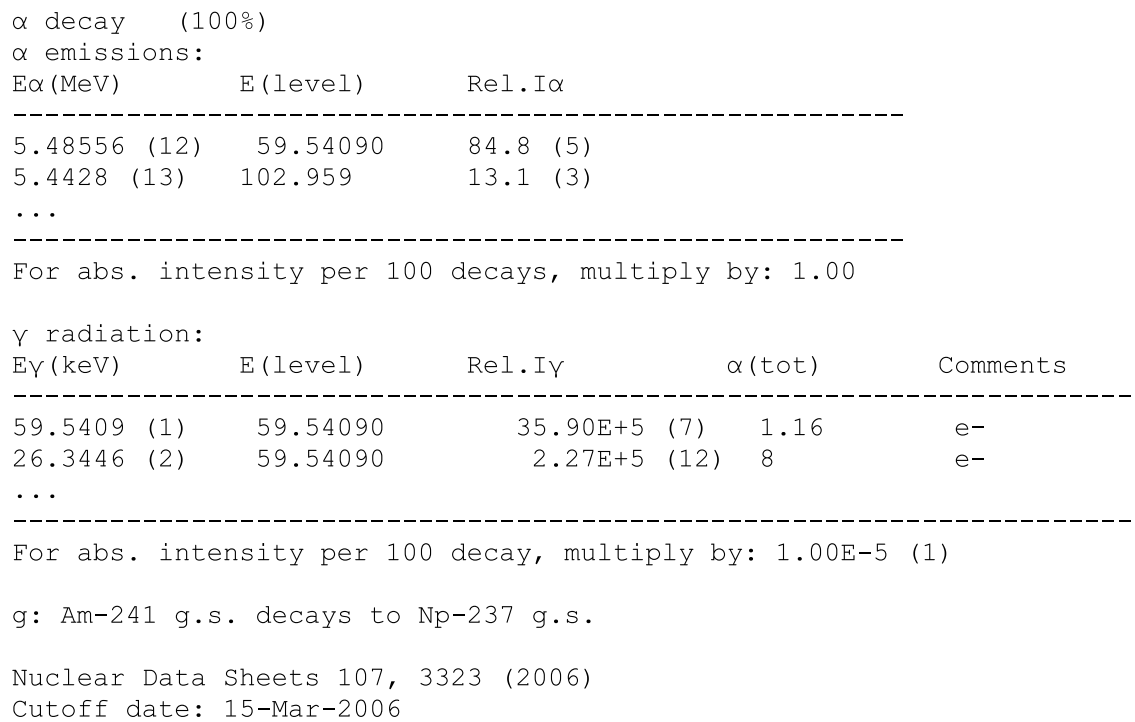

Fig. 5. References for decay data for individual nuclides are available in the online version of the Karlsruhe Nuclide Chart. In this example for Am 241 note the data sources for half-life and decay modes/radiations are from different NDS evaluations.

depending on which is the more recent. In cases where the data may be incomplete, the Nuclear Science References [6] are consulted to find the original literature.

\subsubsection{Creation and maintenance of scientific references}

Although references for individual nuclides are not given in the printed versions, they are available in the online version (Karlsruhe Nuclide Chart Online). An example of such a reference for an individual nuclide is shown in Figure 5 for Am 241. The most recent evaluation of mass number 241 was in 2015 (NDS Vol. 130). There the half-life of Am 241 was updated but not the decay and radiation data (since this latter data is associated with the daughter products). The most recent evaluation of the decay data (with mass 237 ) in ENSDF was in 2006 (NDS Vol. 107). This is the reason why two references are given in Figure 5. For more recent references, a hyperlink to the journal is given as shown. The Q-value was checked as described above. Following the introduction of this procedure in 2011, around 2400 ground and isomeric states have been referenced in this manner.

\section{Other data in the nuclide chart}

\subsection{Isotopic abundances}

The isotopic abundance of an isotope refers to the relative proportion of that isotope to the stable or primordial isotopes of that element in terrestrial matter. Isotopic abundances are expressed in atom $\%$ and are given in the nuclide boxes for stable and for primordial isotopes of a 


\begin{tabular}{|c|c|c|c|c|c|c|c|c|}
\hline $\begin{array}{c}\text { O 13 } \\
8.58 \mathrm{~ms} \\
\\
\beta^{+} 16.7 \ldots \\
\beta p(1.44,6.44 \ldots \\
\gamma\left(4439^{*}, 3500 \ldots\right)\end{array}$ & 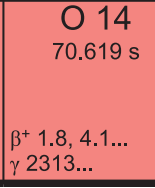 & 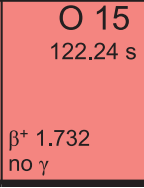 & $\begin{array}{l}\text { O } 16 \\
99.757\end{array}$ & \begin{tabular}{|r}
017 \\
0.038 \\
\\
\\
$\sigma 0.00054$ \\
$\sigma_{n, \alpha} 0.257$ \\
\end{tabular} & $\begin{array}{l}18 \\
0.205\end{array}$ & $\begin{array}{r}019 \\
26.476 \mathrm{~s} \\
\\
\beta \beta \\
\beta-3.3,4.8 \ldots \\
\gamma 197,1357 \ldots \\
\end{array}$ & $\begin{array}{l}\mathrm{O} 20 \\
13.5 \mathrm{~s}\end{array}$ & $\begin{array}{l}\text { O } 21 \\
\quad 3.42 \mathrm{~ms} \\
\\
\\
\beta-6.4 \ldots \\
\gamma \gamma 1730,3517 \\
280,1787 \\
1755 \ldots\end{array}$ \\
\hline
\end{tabular}

Fig. 6. The element oxygen $(\mathrm{O})$ has 15 isotopes in the 10th edition of the Karlsruhe Nuclide Chart. In the above figure, 9 of these isotopes are shown. The three stable isotopes: O 16, O 17 and O 18 are shown in black. The natural isotopic abundances are respectively: $99.757,0.038$ and 0.205 atom $\%$.

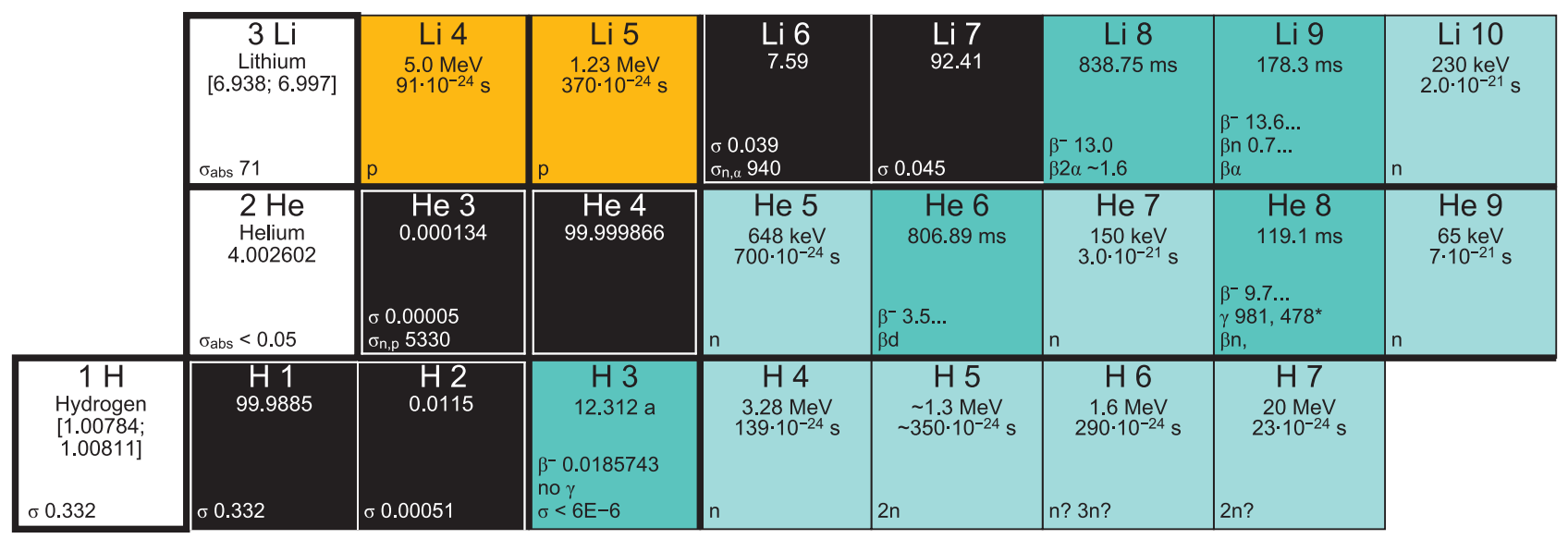

Fig. 7. Excerpt from the 10th edition of the Karlsruhe Nuclide Chart. The three horizontal lines show isotopes of elements H, He and $\mathrm{Li}$. The white boxes represent the chemical element and contain the standard atomic weights and thermal neutron cross sections for the element.

chemical element. Figure 6 shows an excerpt from the Karlsruhe Nuclide Chart showing stable (black) and unstable (red/blue) isotopes of oxygen. In the difference chart (Fig. 3), the black coloured boxes denote the nuclides with isotopic abundances modified in the latest edition. The isotopic abundances were taken from the most recent evaluations i.e. Isotopic Composition of Elements 2013 and 2009 (IUPAC Technical Reports) [7,8].

\subsection{Thermal neutron cross sections}

All cross sections (symbol $\sigma$ ) are given in barn $\left(10^{-24} \mathrm{~cm}^{2}\right)$ and refer to reactions with thermal neutrons with energy $0.0253 \mathrm{eV}$. The symbol $\sigma$ without any index in the nuclide boxes refers to the $(\mathrm{n}, \gamma)$ capture cross section. If two values are given, the first refers to the formation of the product nucleus in the metastable, the second to the formation in ground state. The symbols $\sigma_{\mathrm{f}}, \sigma_{\mathrm{n}, \mathrm{p}}, \sigma_{\mathrm{n}, \alpha}$ and $\sigma_{\mathrm{abs}}$ refer to the fission, (n,p), (n, $\alpha)$ and absorption cross sections respectively. In Figure 6, values following the symbol $\sigma$ in boxes O $16, \mathrm{O}$ 17 and $\mathrm{O} 18$ are the cross sections for $(\mathrm{n}, \gamma)$ reactions for the formation of nuclides O 17, O 18 and O 19, respectively. The value $\sigma_{\mathrm{n}, \alpha}$ in the box $\mathrm{O} 17$ is the cross section of the reaction for formation of carbon $\mathrm{C} 14$ isotope. The cross section values used in the 10th edition are from the CRC Handbook of Chemistry and Physics 97 Ed. 2016-2017 [9].

\subsection{Standard atomic weights}

Each horizontal line (with constant Z, variable N) in the twodimensional nuclide chart contains the isotopes of a particular chemical element (Fig. 7). In each horizontal line, the proton number is fixed and only the neutron number varies. At the start of each line, the white box represents the element. Directly below the chemical symbol and the name the standard atomic weight is given based on the assumption that the mass of the $\mathrm{C} 12$ isotope equals 12 atomic mass units. For twelve elements H, Li, B, C, N, O, Mg, Si, S, Cl, Br and Tl, a range of atomic weights is given to reflect the isotopic variability in the natural materials. The atomic weight data has been taken from atomic weights of the elements 2013 (IUPAC Technical Report) [10].

\subsection{Chain yields for thermal neutron fission of $U 235$ and $\mathrm{Pu} 239$}

The thermal neutron chain yields of the two important fissile nuclides U 235 and $\mathrm{Pu} 239$ are shown to the right of the nuclide chart, at the end of the isobar (Fig. 8). The upper values correspond to U 235 and the lower values to $\mathrm{Pu} 239$ thermal neutron fission yields. The fission yields are taken from: IAEA Handbook of Nuclear Safeguards from 2008 [11]. This IAEA evaluation gives data recommended by international working groups and it contains data also for ternary fissions. Additional chain yields, not given in the IAEA report have been taken from the JEFF 3.1 Nuclear Data Library (2006) [12]

\subsection{Physical constants}

In the booklet accompanying the Karlsruhe Nuclide Chart, a list of important physical constants and conversion factors is given. In the new edition of the Chart, the values 


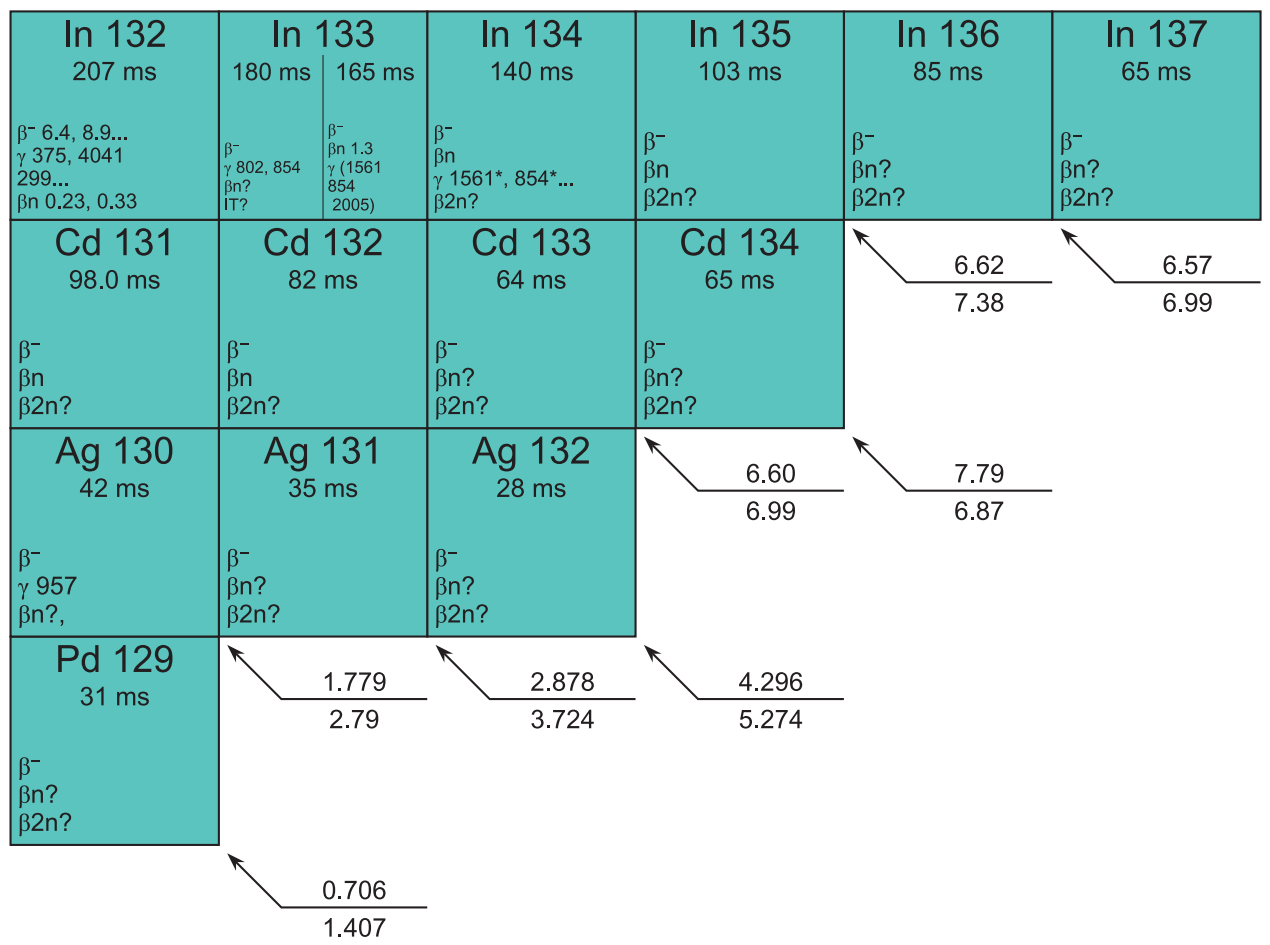

Fig. 8. Mass chain fission yields for masses 129-136. The upper and lower values are for thermal fission of U 235 and Pu 239 , respectively.

were updated based on the CODATA recommended values of the fundamental physical constants [13].

\subsection{Properties of the elements and the periodic table of elements}

The booklet also provides the most important properties of chemical elements in a tabular form. The properties include allotropic form, density, triple point, melting point, boiling point, critical point and ionization potential. Data were taken from latest editions of the CRC Handbook of Chemistry and Physics [9] except densities of gaseous elements which were published only in a previous edition of this handbook [14]. On the last pages of the booklet a Periodic Table of the Elements based on the latest IUPAC data is given.

\section{Decay and nuclear reactions in Karlsruhe Nuclide Chart}

The Karlsruhe Nuclide Chart can be used to investigate decay and nuclear reactions as shown in the nuclide chart sections in Figures 9a and 9b. In Figure 9a, the location of the daughter products following decay by $\alpha, \beta^{-}, \varepsilon / \beta^{+}, n$, and $\mathrm{p}$ decay processes are shown. In $\alpha$ emission for example, the parent nuclide loses two protons and two neutrons. Hence, this results in the daughter position shown in Figure 9a. In $\beta^{-}$emission, the parent loses one neutron and gains one proton as shown in Figure 9a. With these "rules" for decay processes, entire decay chains can be obtained manually. An example of this is given in Figure 10 for the decay of Th 232. The daughter products are shown highlighted. The parent nuclide Th 232 decays by alpha emission to Ra 228. This decays further through $\beta^{-}$emission to Ac 228, and so on.

Similarly, the nuclide chart can be used to obtain activation and nuclear reaction products. This is shown schematically in Figure 9b. A target nuclide with $\mathrm{Z}$ protons and $\mathrm{N}$ neutrons will transform to a reaction product through the process shown. In the case of a $(n, \gamma)$ reaction (capture reaction), the target nuclide absorbs a neutron. The excess energy in the activation process is released via gamma emission. The resulting product contains one neutron more than the original target nuclide. Again with these simple rules, the entire build-up of activation products can be established using the Chart. In a nuclear reactor U 238, for example, transforms to $\mathrm{U} 239$ through $(\mathrm{n}, \gamma)$ reactions. The $\mathrm{U}$ 239 then decays through $\beta^{-}$emission to produce $\mathrm{Np} 239$, etc.

\section{Printed and online editions of the Chart}

\subsection{Print editions}

The Karlsruhe Nuclide Chart is published in a number of printed and online editions. The printed versions are available in various formats:

- a fold-out chart in A4 format;

- a wall-chart in a poster format $(100 \times 139 \mathrm{~cm})$ in which the full Chart is divided into 4 branches for compactness.

A larger version of this is also available $(120 \times 170 \mathrm{~cm})$; - an auditorium chart with the entire nuclide chart in a single section $(43 \times 316 \mathrm{~cm})$.

In each case, the Chart is accompanied by a 72 page booklet/brochure (see https://nuklid.shop). 


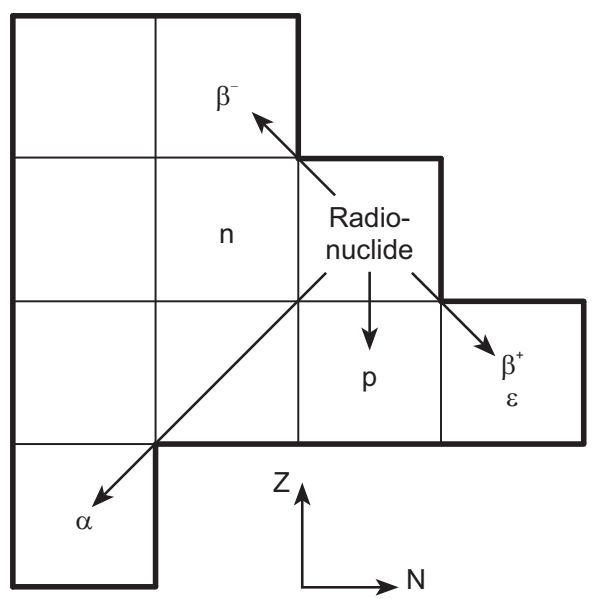

(a)

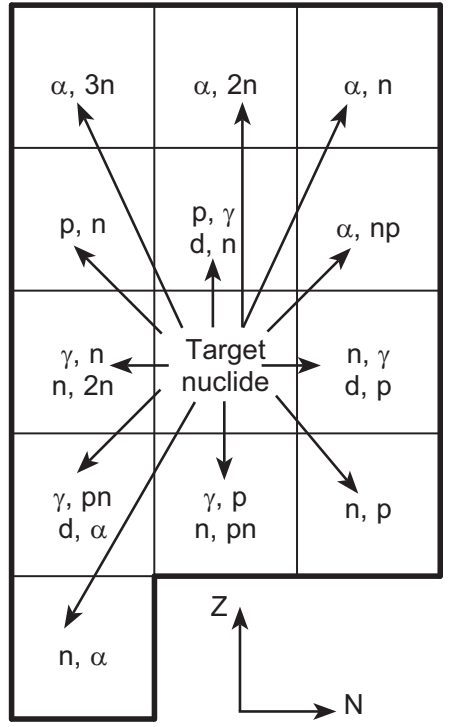

(b)

Fig. 9. (a) Radioactive decay processes on the nuclide chart. A "parent" with coordinates Z, N transforms to the "daughter" product through the decay process shown. (b) Activation processes and nuclear reactions on the nuclide chart. A target nuclide with

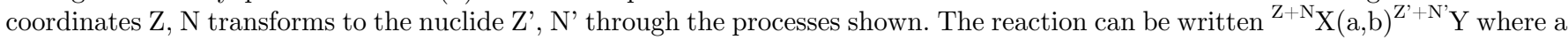
and $\mathrm{b}$ are the incident and exiting particles respectively.

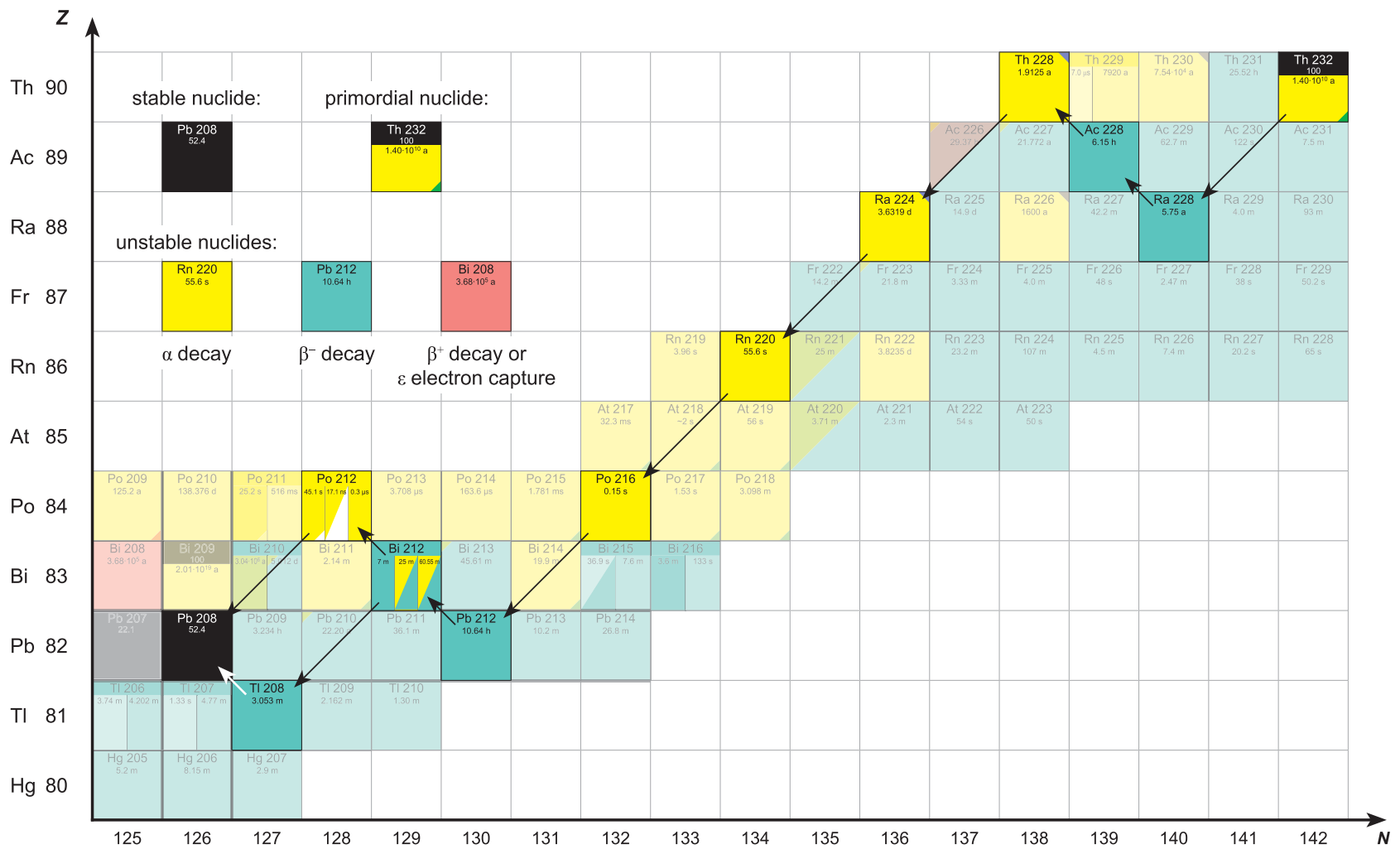

Fig. 10. Highlighted decay chain of Th 232 showing the parent and successive daughters. 
In addition to the above formats, customised formats are also available (for more information see http://www. karlsruhenuclidechart.com).

\subsection{Online editions}

In addition to the print editions, online editions of the Karlsruhe Nuclide Chart are also available through the nuclear science portal Nucleonica (ref.: https://nucleonica. com). The online versions have a number of additional features which are not available in the printed versions:

- colour coding based on half-lives and the modes of decay; - access to current and earlier editions of the Charts (7th edition 2006, 8th edition 2012, 9th edition 2015, 10th edition 2018), together with statistics on the number of nuclides and updates in each edition;

- fast navigation through more than 4000 nuclide ground states and isomers. Nuclide searching based on half-lives; energies and emission probabilities of gamma and X-rays, alpha, $\beta^{-}$, ec $/ \beta^{+}$radiations, etc.;

- scientific references for new and updated nuclides.

Both the printed and online editions of the Karlsruhe Nuclide Chart are restricted to the provision of key nuclear decay and thermophysical data for a multi-disciplinary audience. For a more intensive use of this data in scientific applications, the user is referred to the Nucleonica nuclear science portal. There validated scientific applications for many types of calculations are provided including decay calculations, dosimetry and shielding, nuclide datasheets, gamma spectrometry, range and stopping power, transport and packaging, together with detailed descriptions on the use of these applications.

\section{Conclusions}

The Karlsruhe Nuclide Chart is a widely used tool in a broad range of scientific disciplines ranging from traditional physical sciences to life and earth sciences and healthcare. From the highly detailed information provided by data scientists worldwide, the Chart identifies and provides the key nuclear data in a form required and understandable to non-specialists.

In this paper, the scientific highlights of the 10th Edition (2018) of the Karlsruhe Nuclide Chart are presented. This edition coincides with the 60th anniversary of the Chart which was first published in 1958. The new edition of the Chart contains nuclear data on 4040 experimentally observed ground states and isomers and covers half-lives, branching ratios, decay modes, and energies of the emitted radiations. Since the previous edition in 2015, 649 nuclides have been updated and 47 new nuclides identified. The new IUPAC names nihonium, moscovium, tennessine and oganesson for elements 113, 115,117 , and 118 respectively have been incorporated.

The paper also provides a description of the Karlsruhe Nuclide Chart with particular focus on the structure of the nuclide boxes and how this data can be related to the decay schemes. Details of decay data used are given including a dedicated section on data sources used, the update procedure, and nuclide specific scientific references. A section of the paper is devoted to explaining how the Chart can be used to investigate decay chains and nuclear reactions.

Finally, the paper summarises additional data provided with the Karlsruhe Nuclide Chart including the latest literature values for isotopic abundances, thermal neutron cross sections, standard atomic weights, chain yields for thermal fission of $\mathrm{U} 235$ and $\mathrm{Pu} 239$, physical constants, and properties of the chemical elements. The Chart also provides a multi-lingual "Explanation of the Chart of the Nuclides" in English, German, French, Spanish, Russian, and Chinese for an international audience. Finally, the paper provides information on the various printed and online editions of the Karlsruhe Nuclide Chart available.

Figures presented in this paper are extracted from the Karlsruhe Nuclide Chart and Karlsruhe Nuclide Chart Online courtesy of Nucleonica GmbH.

\section{Author contribution statement}

All the authors have contributed with selection and visualisation of scientific nuclear data for Karlsruhe Nuclide Chart. Writing the paper, the technical review and the incorporation of the referees' requirements were also collaborative tasks of all three authors.

\section{References}

1. L. Ohrstrom, J. Reedijk, Names and symbols of the elements with atomic numbers 113, 115, 117 and 118 (IUPAC Recommendations 2016), Pure Appl. Chem. 88, 1225 (2016)

2. E.A. McCutchan, ed., Mass Chain Evaluations 2015-2017, Nuclear Data Sheets (Elsevier, Amsterdam, NL, issues between 2015-2017), available at: https://www.sciencedir ect.com/journal/nuclear-data-sheets/issues

3. Evaluated Nuclear Structure Data File (ENSDF), Online Data Services, ed.: National Nuclear Data Center at Brookhaven National Laboratory, US, https://www.nndc. bnl.gov/ensdf/

4. J. Magill, G. Pfennig, R. Dreher, Z. Sóti, Karlsruhe Chart of the Nuclides 9th Edition, ISBN: 978-943868-04-3 (Nucleonica GmbH, Germany, 2015)

5. G. Audi, F.G. Kondev, M. Wang, W.J. Huang, S. Naimi, The NUBASE2016 evaluation of nuclear properties, Chin. Phys. C 41, 030001 (2017)

6. B. Pritychenko, E. Běták, M.A. Kellett, B. Singh, J. Totansa, The Nuclear Science References (NSR) database and Web Retrieval System, Nucle. Instrum. Methods Phys. Res. A 640, 213 (2011)

7. J. Meija, T.B. Coplen, M. Berglund, W.A. Brand, P.D. Bièvre, M. Gröning et al., Isotopic compositions of the elements 2013, Pure Appl. Chem. 88, 293 (2016)

8. M. Berglund, M.E. Wieser, Isotopic compositions of the elements 2009, Pure Appl. Chem. 83, 397 (2011) 
9. W.M. Haynes, ed., CRC Handbook of Chemistry and Physics, 97th Edition (CRC Press, Boca Raton, Florida, US, 2016-2017)

10. J. Meija, T.B. Coplen, M. Berglund, W.A. Brand, P.D. Bièvre, M. Gröning et al., Atomic weights of the elements 2013, Pure Appl. Chem. 88, 265 (2016)

11. A.L. Nichols, D.L. Aldama, M. Verpelli, Handbook of nuclear data for safeguards: Database extensions, IAEA Report INDC(NDS)-0534 (International Atomic Energy Agency, Vienna, Austria, 2008)
12. A. Koning, R. Forrest, M. Kellett, R. Mills, H. Henriksson, Y. Rugama et al., The JEFF 3.1 Nuclear Data Library-JEFF Report 21, OECD/NEA (Nuclear Energy Agency of the OECD (NEA), Paris, France, 2006)

13. P.J. Mohr, D.B. Newell, B.N. Taylor, CODATA recommended values of the fundamental physical constants: 2014, J. Phys. Chem. Ref. Data 45, 043102 (2016)

14. W.M. Haynes, ed., CRC Handbook of Chemistry and Physics, 95th Edition (CRC Press, Boca Raton, Florida, US, 2014-2015)

Cite this article as: Zsolt Sóti, Joseph Magill, Raymond Dreher, Karlsruhe Nuclide Chart- New 10th edition 2018 , EPJ Nuclear Sci. Technol. 5, 6 (2019) 